\section{How alum works}

For more than 70 years, alum has been used as a trusty aide to improve antibody responses to vaccines. But how this aluminum-containing compound boosts the response to vaccines has been a mystery. On page 869, Kool et al. expose its mysterious mechanism; they find that alum causes cells to produce a stimulator of dendritic cells (DCs).

DCs were once the favored hypothetical link between alum and B cells, as they activate $\mathrm{CD} 4^{+} \mathrm{T}$ cells, which can then enhance $\mathrm{B}$ cell activation and antibody production. But DCs were later dismissed when it was found that they were not stimulated by alum in vitro.

These in vitro results are now shown to be a red herring by Kool et al., who find that alum activates DCs in vivo by provoking the secretion of uric acida molecule that is triggered by tissue and cell trauma. The injection of alum, the group found, induced an influx of neutrophils and inflammatory cytokines and chemokines - a combination that was previously seen in response to the injection of uric acid into mice.

In mice injected with antigens mixed with alum, uric acid levels increased within hours. The uric acid might be released by the cells' lining the body's cavities that turn necrotic after contacting the alum. The absence of these uric acid sources in in vitro assays might have led to the previous misleading results.

In response to the uric acid, inflammatory monocytes flocked to the injection site, took up the antigens, and broke them down into $\mathrm{T}$ cell-stimulating epitopes. The monocytes then migrated to lymph nodes, where they matured into DCs and activated $\mathrm{CD} 4^{+} \mathrm{T}$ cells.

Without alum, the antigens were not taken up at the injection site. Still, they eventually reached lymph nodes via the flowing lymph. The resident node DCs,

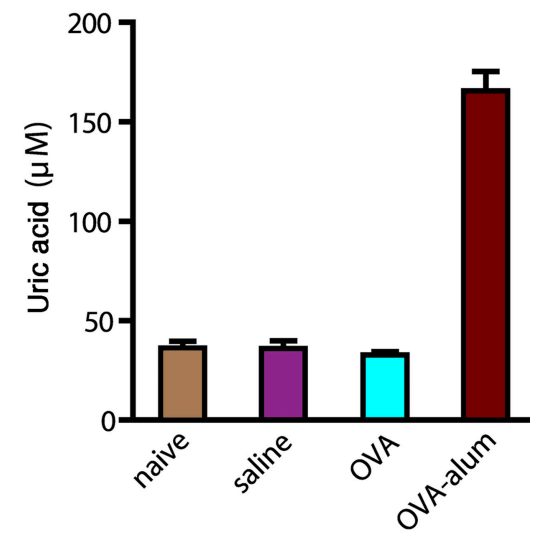

Mice injected with antigens mixed with alum produce uric acid that then attracts monocytes.

however, did not process the alum-free antigens efficiently or express $\mathrm{T}$ cell costimulating receptors. The resulting subdued immunity was similar to that seen in mice that were depleted of inflammatory monocytes or those injected with enzymes that degrade uric acid. JEM

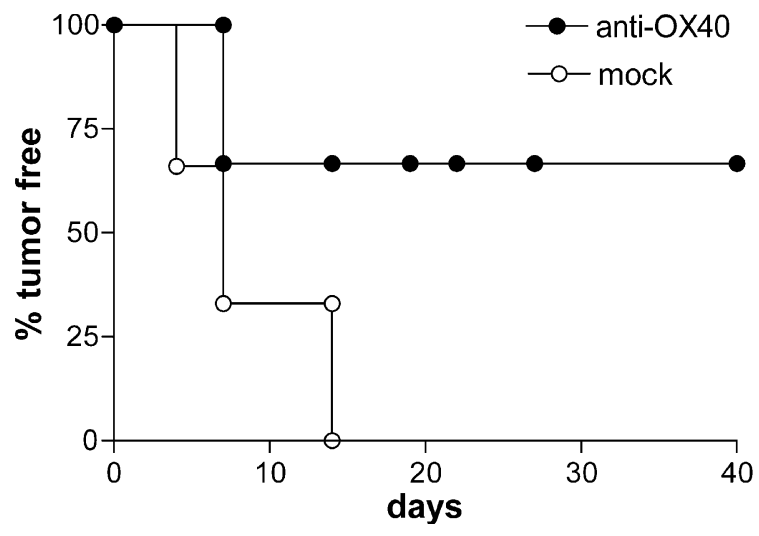

Mice injected with agonist anti-0X40 antibody remain tumorfree after injection with cancer cells.

\section{OX40: a win-win path to tumor immunity}

Regulatory $T$ ( $T$ reg) cells prevent autoimmunity by keeping self-reactive effector T cells in check, but this suppression becomes counterproductive when the effector cells are prevented from attacking tumors. Piconese et al. now show on page 825 , however, that tumor immunity can be achieved without risking autoimmunity simply by stimulating a T cell surface protein called OX40.

A major challenge in tumor immunotherapy lies in breaking $T$ reg cell-mediated tumor tolerance without inducing organism-wide autoimmunity or compromising immune surveillance. This goal has remained elusive because current methods used to derail T reg cell activity-such as depleting them using antibodies to a major T reg cell surface marker, CD25-also target effector T cells, thereby preventing tumor immunity and perhaps immunity to pathogens. T reg cell depletion also provokes conversion of effector T cells into T reg cells, thus worsening the initial immune suppression.

Piconese et al. sought a different approach by targeting OX40 because its stimulation suppresses T reg cell function while enhancing effector T cell survival and activity in vitro. Mice injected with agonistic anti-0X40 antibody before their injection with a carcinoma cell line efficiently rejected the tumor. Anti-0X40 injection also melted established tumors and promoted lasting immunity against the cancer.

Suppression of T reg cells in the tumor enhanced the migration of tumor-infiltrating dendritic cells (DCs) and allowed the DCs to carry tumor antigens to the draining lymph node, where they could then activate a new wave of tumor-reactive T cells. Meanwhile, the triggering of OX40 on effector T cells also enhanced tumor immunity, thus providing a double benefit. Mice injected twice with anti-0X40 antibody showed no evidence of autoimmunity and no change in T reg cell number, so OX40 may be a promising target for cancer immunotherapy. JEM 